\title{
Chitinase 3-like-1 enhances bacterial adhesion to colonic epithelial cells through the interaction with bacterial chitin-binding protein
}

\author{
Mayumi Kawada ${ }^{1,2}$, Chun-Chuan Chen ${ }^{1}$, Atsuko Arihiro ${ }^{1}$, Katsuya Nagatani ${ }^{1}$, Takeshi Watanabe ${ }^{3}$ and \\ Emiko Mizoguchi ${ }^{1,2}$
}

Dysregulated host/microbial interactions play a pivotal role in the pathogenesis of inflammatory bowel disease. We previously reported that chitinase 3-like-1 (CHI3L1) enhances bacterial adhesion and invasion on/into colonic epithelial cells (CECs). In this study, we designed to identify the exact mechanism of how CHI3L1 enhances the bacterial adhesion on CECs in vitro. As compared with wild type (WT) of Serratia marcescens, chitin binding protein (CBP) 21 knockout strain of S. marcescens significantly decreased the adhesion to SW480 cells that express CHI3L1 endogenously. A CBP21 fusion protein was produced with CBP21-expressing vector, which was transformed into BL21 strain of Escherichia coli. CBP21 overexpression significantly increased the adhesion, but not invasion, of nonpathogenic $E$. coli. The adhesion of

S. marcescens and CBP21-overexpressing E. coli was inhibited by coculture with chitin, but not with other carbohydrates. After overexpressing CHI3L1 on SW480 cells, the adhesion rate of CBP21-overexpressing E. coli was further increased by approximately twofold. Genetically engineered $E$. coli with a single mutation of either Thy- 54 or Glu-55 position of CBP21 exhibited a decreased binding ability, and the binding was $74 \%$ diminished by the combined mutations of three amino acids (Thy-54, Glu-55 and Glu-60) as compared with WT. Inhibition of CHI3L1 by anti-CHI3L1 antibody or CHI3L1-specific short interfering RNA reduced the adhesion of CBP21-overexpressing E. coli to CECs. In conclusion, CHI3L1 is involved in the enhancement of CBP-expressing bacterial adhesion to CECs. CBP21 and its homologs may be required for the CHI3L1mediated enhancement of bacterial adhesion to CECs through the conserved amino-acid residues.

Laboratory Investigation (2008) 88, 883-895; doi:10.1038/labinvest.2008.47; published online 19 May 2008

KEYWORDS: inflammatory bowel disease; chitinase 3-like-1; chitin binding protein; bacterial adhesion; colonic epithelial cells; Serratia marcescens

Chitin, a polymer of $\beta$-1, $4-N$-acetyl-glucosamine (GlcNAc) and the second most abundant polysaccharide in nature next to cellulose, is produced by almost all living organisms, including insects, fungi, crustaceans, and other marine organisms, but not by mammals. ${ }^{1,2}$ Chitin is degraded by chitinases (EC 3.2.1.14) that belong to members of the glycoside hydrolase family 18 . Chitinases are generally considered to be lacking in mammalian bodies due to the absence of chitin. However, recent studies have identified that chitinases and chitinase-like proteins belonging to the glycoside hydrolase family 18 actually exist in mice and humans. The members of mammalian chitinases and chitinase-like proteins include chitinase 3-like-1 (CHI3L1; also known as YKL-40 or human cartilage glycoprotein $39(\mathrm{HCgp}-39)){ }^{3}$ chitotriosidase, ${ }^{4}$
YKL-39, ${ }^{5}$ Ym1, ${ }^{6}$ acidic mammalian chitinase (AMCase), ${ }^{7}$ oviduct-specific glycoprotein ${ }^{8}$ and stabilin-1-interacting chitinase-like protein. ${ }^{9}$

Chitinase 3 -like-1 is a $40-\mathrm{kDa}$ mammalian glycoprotein $^{8,10,11}$ and is produced by human synovial cells, ${ }^{12}$ osteosarcoma cells, ${ }^{10}$ chondrocytes, ${ }^{3,13}$ smooth muscle cells, ${ }^{14}$ macrophages, ${ }^{15,16}$ neutrophils, ${ }^{17}$ colonic epithelial cells $(\mathrm{CECs})^{18}$ and others. AMCase and chitotriosidase have catalytic activity, but the other chitinases including CHI3L1 do not possess the enzymatic activity. ${ }^{9}$ Due to the substitution of an essential glutamic acid residue to leucine, CHI3L1 has no chitinase activity, ${ }^{3}$ but still can bind chitin and chitooligosaccharides with high affinity through a preserved hydrophobic substrate binding cleft. ${ }^{16,19}$ Previously, we

\footnotetext{
${ }^{1}$ Gastrointestinal Unit, Department of Medicine, Boston, MA, USA; ${ }^{2}$ Center for the Study of Inflammatory Bowel Disease, Massachusetts General Hospital, Harvard Medical School, Boston, MA, USA; ${ }^{3}$ Department of Applied Biological Chemistry, Faculty of Agriculture, Niigata University, Niigata, Japan Correspondence: Dr E Mizoguchi, MD, PhD, Gastrointestinal Unit, GRJ-702, Massachusetts General Hospital, 55 Fruit Street, Boston, MA 02114, USA.

E-mail: emizoguchi@partners.org

Received 29 October 2007; revised 04 April 2008; accepted 05 April 2008
} 
demonstrated that the expression of CHI3L1 molecule is highly induced in CECs and macrophages with inflammation and enhances potentially pathogenic bacterial adhesion and invasion on/into CECs. ${ }^{18}$

Suzuki et $a l^{20}$ first identified that a gram-negative bacterium, S. marcescens, produces a chitin-binding protein, CBP21, in the culture supernatant, which enhances chitin accessibility to bacterial chitinases and cloned the corresponding gene. A multiple alignment of bacterial chitinbinding proteins (CBPs) revealed that 40 highly conserved residues ( $>90 \%$ conserved) and a cluster of conserved, mainly hydrophilic residues exist at the surface of CBP21 molecule. ${ }^{21}$ More importantly, the majority of chitinaseproducing pathogenic microorganisms contain a gene encoding for the homolog of the $\operatorname{cbp} 21$ gene, suggesting the presence of a potential binding ability of chitinase-producing pathogenic bacteria to chitin via CBP21 homolog. ${ }^{22}$

In this study, we propose a new possibility that CHI3L1 expressed on CECs binds to bacterial CBP21 homolog via chitin or chitin-like molecule, and this binding subsequently enhances the adhesion of these bacteria to CECs. To identify the exact mechanism of how CHI3L1 enhances the bacterial adhesion on CECs in vitro, we utilize $S$. marcescens wild type (WT) in its mutant form and CBP21-overexpressing nonpathogenic E. coli.

\section{MATERIALS AND METHODS Cell Culture}

SW480, CMT93 and COS7 cells were obtained from the American Type Culture Collection (ATCC, Manassas, VA, USA) and were cultured in Dulbecco's modified Eagle medium with L-glutamine (Cellgro, Lawrence, KS), supplemented with $10 \%(\mathrm{vol} / \mathrm{vol})$ fetal calf serum and a mixture of antibiotics (penicillin $G$ and streptomycin) (Cellgro).

\section{Bacterial Strains and Culture Condition}

Serratia marcescens strains (ATCC) and BL21-AI strain of E. coli (Invitrogen, Carlsbad, CA, USA) were purchased. All strains were grown in Luria-Bertani broth with shaking at 220 r.p.m. at $37^{\circ} \mathrm{C}$. pRSET bacterial expression kit from Invitrogen was used following the manufacturer's instructions. Briefly, after 1 or $2 \mathrm{~h}$ of incubation with $1 \mathrm{mM}$ isopropyl $\beta$-Dthiogalactoside (IPTG) (Sigma, St Louis, MO, USA) and $0.2 \%$ L-arabinose (Sigma), BL21-AI strains of E. coli were spun down and resuspended in $100 \mu \mathrm{l}$ of phosphate-buffered saline (PBS) and subjected to repeated freeze-thaw cycles to extract the insoluble protein. After centrifugation at 13000 r.p.m., the supernatant was electrophoresed on an SDS-polyacrylamide gel and stained with Coomassie blue. For adhesion and invasion assays, BL21-AI strains of E. coli were incubated with $1 \mathrm{mM}$ IPTG and $0.2 \% \mathrm{~L}$-arabinose for $2 \mathrm{~h}$ according to the manufacturer's instruction. After the incubation, E. coli were washed and resuspended in the same volume of PBS and added to the cells. As internal controls, we used pRSET/B empty vector (Invitrogen) or pRSET/B lacZ-expressing E. coli (Invitrogen) in the study.

\section{Generation of CBP21 Knockout Strain of S. marcescens}

The internal region (320 bp) of the $c b p$ gene was amplified by PCR with a primer set, Dcbp-F (5'-catgttcggcgtttcgcaac- $\left.3^{\prime}\right)$ and Dcbp-R ( $5^{\prime}$-tgatgaaatagcgccagctg- $\left.3^{\prime}\right)$, and chromosomal DNA of S. marcescens 2170 was used as a template. The amplified fragment was ligated with SmaI-cut pUC119 to generate plasmid pUCDCBP, and nucleotide sequence was confirmed. The amplified fragment was digested out with $\mathrm{XbaI}$ and SacI from pUCDCBP and ligated with $\mathrm{XbaI}$ and SacI-cut pFS200. Obtained plasmid, pFSCBP, carrying a portion of the $c b p$ gene was first introduced into $E$. coli strain S17-1 (lpir) by electroporation and then transferred to S. marcescens 2170 by conjugation as described previously. ${ }^{23}$ Transconjugants containing pFSDCBP in the $c b p$ gene on the chromosome were selected on Luria-Bertani agar plates containing chloramphenicol $(100 \mu \mathrm{g} / \mathrm{ml})$ and tetracycline $(25 \mu \mathrm{g} / \mathrm{ml})$.

\section{Complementation of CBP21 Expression in CBP21 Knockout Strain of S. marcescens}

For complementation of CBP21-negative S. marcescens, a polyvalent expression vector, pMMB67EH (ATCC 37622) was used as previously described. ${ }^{24}$ Briefly, full-length CBP21 cDNA inserted PCR2.1-TOPO vector (Invitrogen) was excised by EcoRI and ligated into PMMB67EH (CBP21PMMB67EH). CBP21-PMMB67EH was transformed into E. coli HB101 containing helper plasmid pRK2013 (ATCC 37159). After confirming the sequence, this strain was then conjugated with CBP21 knockout strain of S. marcescens. Colonies of CBP21-overexpressing CBP21 knockout strain were isolated under the restriction of tetracycline $(20 \mu \mathrm{g} / \mathrm{ml})$ and ampicillin $(100 \mu \mathrm{g} / \mathrm{ml})$, which CBP21 knockout strain of S. marcescens and CBP21-PMMB67EH are resistant to, respectively.

\section{Generation of CBP21-Overexpressing Nonpathogenic E. coli}

Full length (549 bp) of CBP21 cDNA was cloned into the TOPO 2.1 TA cloning vector (Invitrogen). After confirmation of the sequence, the CBP21 DNA was inserted into the pRSET-B expression vector (Invitrogen), which is a pUCderived expression vector designed for high-level protein expression in nonpathogenic E. coli. The DNA insert of CBP21 was positioned downstream and in frame with a sequence that encodes an $\mathrm{N}$-terminal fusion peptide tagged with polyhistidine (6xHis) and Xpress and transformed into BL21-AI strain of E. coli. All CBP21 mutants were generated by PCR using the QuickChange XL Site-Directed Mutagenesis Kit (Strategene, Cedar Creek, TX, USA) according to the manufacturer's instructions. Point mutations of CBP21 were generated by using the PCR primers as shown in Table 1 . The 
Table 1 Sequences of sense and antisense primers for CBP21 mutants

\begin{tabular}{lll}
\hline Mutations & Sense primers $^{\mathbf{a}}$ & Antisense primers $^{\mathbf{a}}$ \\
\hline Y54A & 5'-CGCAGTGCGGCAGCGTGCAGGCCGACCGCAGAGCGTCGA & 5'-TCGACGCTCTGCGGTTCGGCCTGCACGCTGCCGCACTGCG \\
E55A & 5'-CGGCAGCGTGCAGTACGCACCGCAGAGCGTCGAGGCCTG & 5'-CAGGCCTCGACGCTCTGCGGTGCGTACTGCACGCTGCCG \\
E60A & 5'-CAGTACGAACCGCAGAGCGTCGCAGGCCTGAAAGGCTTCC & 5'-GGAAGCCTTCAGGCCTGCGACGCTCTGCGGTCGTACTG \\
Y54A/E55A/E60A (3MU) & 5'-GCGTGCAGGCCGCACCGCAGAGCGTCGCAGGCCTGAAAGG & 5'-CCTICAGGCCTGCGACGCTCTGCGGTGCGGCCTGC \\
H114A & 5'-CCTGGAAGCTGACCGCCCGTGCCAGCACCACCAGCTGGCG & 5'-CGCCAGCTGGTGGTGTGGCACGGGCGGTCAGCTTCCAGG \\
D182A & 5'-GCCGTGTGGGACATAGCCGCCACCGCCAACGCCTTCTATC & 5'-GATAGAAGGCGTGGCGGTGGGGCTATGTCCCACACGGC \\
N185A & 5'-GGGACATAGCCGACACCGCCGCCGCCTCTATCAGGCGAT & 5'-ATCGCCTGATAGAAGGCGGCGGGGTGTCGGCTATGTC
\end{tabular}

${ }^{\mathrm{a}}$ Mutated areas are shaded, and mutated codons are underlined.

sequence of mutant forms of CBP21 was confirmed at the DNA Core at Massachusetts General Hospital.

\section{Expression Plasmid and Transfection}

The Xpress- and 6xHis-tagged (pCDNA4 His/Max-TOPO) cDNA of mouse CHI3L1 expression vector was described previously. ${ }^{18}$ SW480 and COS7 were seeded at the density of $1-2 \times 10^{5}$ cells/well in 24-well tissue culture plates (Corning Inc., Corning, NY). About $18 \mathrm{~h}$ after seeding the cells, transient transfection was performed using Lipofectamine 2000 (Invitrogen) in SW480 and COS7 cells according to the manufacturer's instructions.

\section{Invasion and Adhesion Assays}

Colonic epithelial cells (SW480, CMT93) were cultured on 24 -well tissue culture plates at a density of $1-2 \times 10^{5}$ cells/ well. After $18 \mathrm{~h}$, transient transfection with CHI3L1 expression vector or pCDNA4 empty vector was performed using Lipofectamine 2000 according to the manufacturer's instructions. Twenty-four hours after transfection, S. marcescens or BL21-AI strains of E. coli were added in each well at a multiplicity of infection (MOI) of 20 and incubated for $1 \mathrm{~h}$ at $37^{\circ} \mathrm{C}$. To determine the total number of cell-associated bacteria, cells were washed with PBS (pH 7.2) three times after the bacterial infection and resolved with $500 \mu \mathrm{l}$ of $1 \%$ Triton X-100 (Sigma) in deionized water for $20 \mathrm{~min}$. The cell lysates were plated on Luria-Bertani agar plates (antibiotic free) for the detection of cell-associated bacteria. Plates were cultured at $37^{\circ} \mathrm{C}$ overnight and colonies were counted on the following day. For invasion assay, after the bacterial infection, cells were washed with the complete culture medium (antibiotics free) and cultured for $1 \mathrm{~h}$ in the presence of $100 \mu \mathrm{g} / \mathrm{ml}$ gentamycin (Invitrogen) in the complete culture medium (antibiotics free) to kill any extracellular bacteria. Cells were washed once with PBS and lysed with $500 \mu \mathrm{l}$ of $1 \%$ Triton $\mathrm{X}-100$ (Sigma) in deionized water, and the bacteria were quantified as described previously. ${ }^{18}$ For the inhibition of CHI3L1 activity, affinity-purified anti-CHI3L1 antibody (Ab), which was generated by the glutaraldehyde conjugation method as a peptide-specific $\mathrm{Ab}$ of mouse CHI3L1 peptide 325-339 amino acid residue, ${ }^{18}$ or purified normal rabbit IgG (Bethyl Laboratories, Montgomery, TX, USA) was added to the cell culture medium $1 \mathrm{~h}$ before bacterial infection and was present through the bacterial infection period. All of the adhesion and invasion assays were performed at least three times in separate experiments.

\section{Adhesion Assay in the Presence of Carbohydrates}

The carbohydrates used in this study were chitin, galactose, mannose, cellulose, and GlcNAc, which were purchased from Sigma. Chitin and cellulose were sonicated for $1 \mathrm{~min}$ and all the carbohydrates were directly dissolved in the appropriate Luria-Bertani or Dulbecco's modified Eagle medium medium at $250 \mu \mathrm{g} / \mathrm{ml}$ final concentration and cocultured overnight with bacteria or CECs.

\section{Confocal Microscopic Analysis}

COS7 cells were seeded on sterile permanox coverslips and were transfected with CHI3L1 construct. After $24 \mathrm{~h}$ of transfection, cells were infected with control-, WT CBP21and 3MU (Y54A, E55A and E60A) CBP21-overexpressing BL21-AI strain of E. coli for $1 \mathrm{~h}$ at an MOI of 20. Cells were washed twice with ice-cold PBS, fixed for $20 \mathrm{~min}$ with cold methanol at $-20^{\circ} \mathrm{C}$, washed three times with ice-cold PBS and blocked for $30 \mathrm{~min}$ with $5 \%$ horse and donkey serum in PBS. Cells were stained using mouse anti-X-press monoclonal $\mathrm{Ab}$ (Invitrogen) at $4^{\circ} \mathrm{C}$ overnight and FITC-conjugated horse anti-mouse IgG (Vector Laboratories, Burlingame, CA, USA) at RT for 1 h. E. coli was detected by rabbit anti-E. coli polyclonal $\mathrm{Ab}$ (DAKO, Carpinteria, CA, USA) at $4^{\circ} \mathrm{C}$ overnight and rhodamine-conjugated anti-rabbit IgG (Jackson ImmunoResearch Laboratories, West Grove, PA, USA) at RT for $1 \mathrm{~h}$. The coverslips were mounted in Vectashield (Vector Laboratories) and examined at RT with a confocal microscope (model Radiance 2000; Bio-Rad Laboratories, Hercules, CA, USA) using multitracking (line switching) for two-color imaging (original magnification: $\times 40$, objective). 
Image acquisition was performed with LaserSharpScanning software (Bio-Rad Laboratories).

\section{Short Interfering RNA}

Three different short interfering RNA (siRNA) duplexes specific for mouse CHI3L1 (gene accession number BC005611) were custom-designed and synthesized by Qiagen (Valencia, CA, USA) as reported previously. ${ }^{18}$ The target of all these three-designed siRNA is located at C-terminal portion of CHI3L1. All the siRNA duplexes were designed using the HiPerformance design algorithm (Qiagen) integrated with a stringent homology analysis tool. The three highestranking siRNA duplexes generated by the algorithm were chosen. The target sequences of CHI3L1 siRNAs are as follows: CHI3L1 siRNA no. 1, AACCCTGTTAAGGAATGCAAA (1416-1436); CHI3L1 siRNA no. 2, CAGCTGTTCAA TAAAGCGCCA (1608-1628); and CHI3L1 siRNA no. 3, CAGGAGTTTAATCTCTTGCAA (1254-1274). As control siRNA, the target sequences are as follows: control siRNA no. 1, AATTCTCCGAACGTGTCACGT; control siRNA no. 2, CGGCAAGCTGACCCTGAAGTTCAT; and control siRNA no. 3, all star negative siRNA (sequence is proprietary for Qiagen). A half $\mu \mathrm{g}$ of each siRNA was transfected to $1 \times 10^{5}$ CMT93 cells/well by RNAiFect Reagent (Qiagen) according to the manufacturer's instructions. Protein expressions of these siRNA effects were examined by western blotting according to the instruction of Invitrogen.

\section{Statistical Analysis}

Statistical significance was evaluated by the Mann-Whitney $U$-test for nonparametric data or the Student's $t$-test for parametric data in this study. $P<0.05$ was considered to be statistically significant.

\section{RESULTS}

\section{Both Adhesion and Invasion Rates of S. marcescens Were} Increased in CHI3L1-Overexpressed CECs

Serratia marcescens is an efficient biological degrader of chitin and one of the most extensively studied chitinolytic bacteria. In addition to chitinases, $S$. marcescens and other chitin-degrading microorganisms secrete CBPs, which bind to chitin with high affinity and specificity. ${ }^{20,25}$ Initially, we performed adhesion and invasion assays of $S$. marcescens Bizio strain (ATCC) to SW480 cells that endogenously expressed CHI3L1. SW480 cells were infected with $S$. marcescens for $1 \mathrm{~h}$ at an MOI of 20 at $37^{\circ} \mathrm{C}$. The adhesion rate of $S$. marcescens relative to original inoculum was $62.5 \pm 5.7 \%$ (mean \pm s.e.m.) (Figure 1a). The invasion rate of $S$. marcescens relative to original inoculum was $0.4 \pm 0.04 \%$ (mean \pm s.e.m.) (Figure 1a). We next transiently transfected with pCDNA4/CHI3L1- or mock (pCDNA4) vector to SW480 cells and subsequently infected the cells with $S$. marcescens. As a result, after being transfected with the CHI3L1 vector to SW480 cells, a significantly increased number of $S$. marcescens bound and invaded into SW480 cells (Figure 1a). These results suggest that
CHI3L1 enhances the adhesion and invasion of $S$. marcescens to CECs.

To examine our hypothesis that bacteria bind to CECs through a CHI3L1/chitin complex, next the effect of chitin on the adhesion efficiency of $S$. marcescens to SW480 cells was determined. For this purpose, pre-incubation of the bacteria or CECs with chitin was performed and followed by an adhesion assay. As shown in Figure 1b, S. marcescens significantly inhibited its binding to CECs after culture with chitin in a dose-dependent manner compared to untreated $S$. marcescens control. In contrast, the adhesion of S. marcescens to SW480 cells was not inhibited when the CECs (instead of bacteria) were pretreated with chitin and the medium was removed before the addition of the inoculum to the cell monolayer (Figure $1 \mathrm{~b}$ ). These data suggest that chitin binds to bacterial side, but not to CECs' side, and chitin efficiently inhibits the bacterial adhesion to CECs.

\section{CBP21 Knockout Strain of S. marcescens Significantly Decreased, but CBP21-Overexpressing CBP21 Knockout Strain Restored, the Adhesion to CECs Compared with WT S. marcescens}

To analyze the biological function of CBP21 molecule in bacterial adhesion to CECs, we generated CBP21 knockout as well as CBP21-overexpressing CBP21 knockout strains of S. marcescens. SW480 cells were infected with WT or CBP21 knockout strain of S. marcescens 2170. Interestingly, CBP21 knockout strain of $S$. marcescens significantly $(P<0.01)$ decreased its adhesion to SW480 cells compared with WT of S. marcescens (Figure 2). In contrast, after CBP21 expression was restored in CBP21 knockout strain of S. marcescens, the bacteria showed a similar binding rate to WT $S$. marcescens (Figure 2). The result strongly suggests a possibility that bacterial CBP21 is an important factor for the adhesion of bacteria to CECs. Therefore, we have focused on analyzing the biological function of bacterial CBPs in bacterial adhesion to CECs.

\section{CBP21-Overexpressing BL21 Strain of E. coli Increased the Adhesion Rate to CECs}

To further examine the functional role of CBPs in bacterial adhesion to CECs, we generated CBP21-overexpressing BL21 strain of $E$. coli transformed with pRSET/B CBP21 (fulllength WT) as described in material and methods. To begin with, we confirmed that recombinant CBP21 fusion protein $(6 \times$ His and Xpress tagged) was produced by E. coli after 1 or $2 \mathrm{~h}$ incubation with $1 \mathrm{mM}$ IPTG detected by SDS-polyacrylamide gel electrophoresis and Coomassie blue stain. As shown in Figure 3a, CBP21 fusion protein can be clearly detected by Coomassie blue stain after $2 \mathrm{~h}$ of incubation with IPTG.

To examine whether CBP21 is involved in the bacterial adhesion, we performed adhesion assays utilizing the nonpathogenic CBP21-overexpressing, pRSET/B empty vector expressing or pRSET/B lacZ-expressing E. coli. SW480 cells 
a

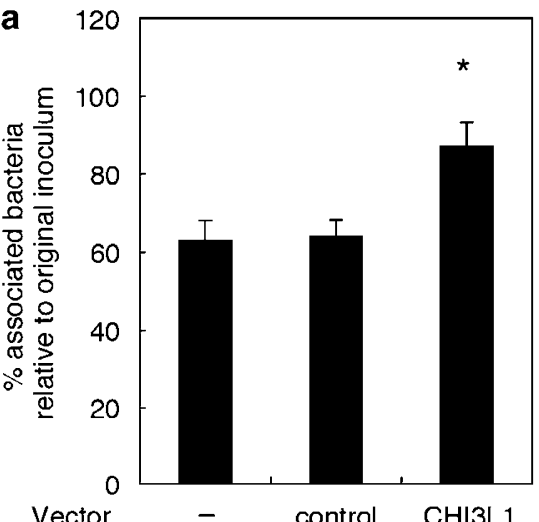

b

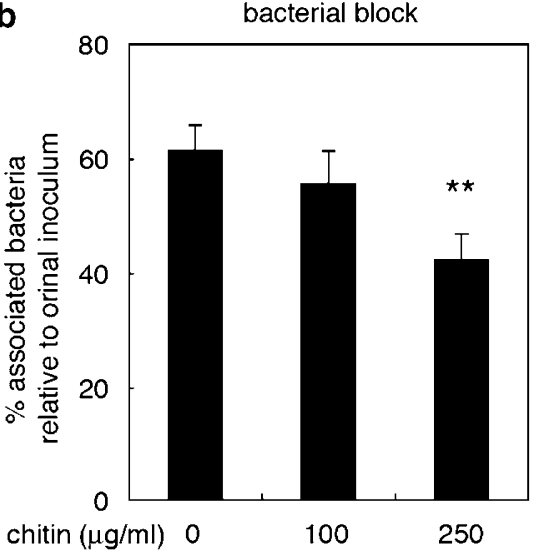

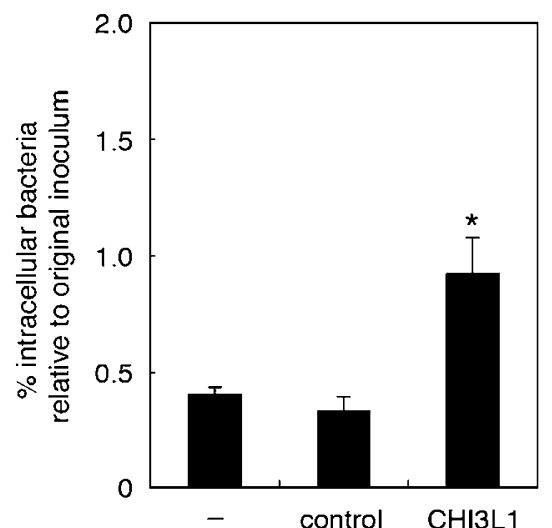

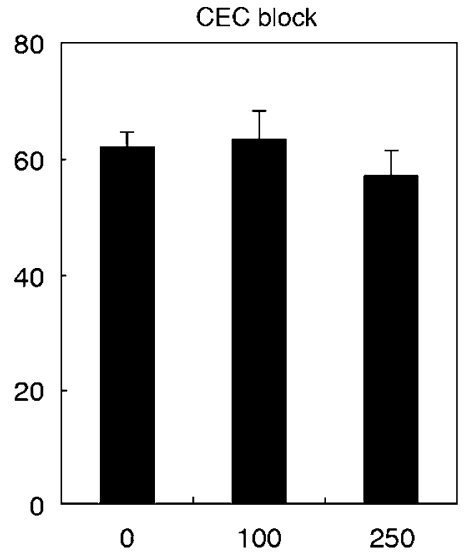

Figure 1 Effect of CHI3L1 and chitin on adhesion and invasion of S. marcescens in CECs. (a) The adhesion and invasion assays of S. marcescens to SW480 cells were performed. SW480 cells were infected with S. marcescens for $1 \mathrm{~h}$ at an $\mathrm{MOI}$ of 20 and incubated at $37^{\circ} \mathrm{C}$. For invasion assay, the cells were cultured for an additional $1 \mathrm{~h}$ with $100 \mu \mathrm{g} / \mathrm{ml}$ gentamycin to kill any extracellular bacteria. For CHI3L1 overexpression, SW480 cells were transfected with pCDNA4/ CHI3L1 vector $(0.5 \mu \mathrm{g})$ or mock pCDNA4 vector $(0.5 \mu \mathrm{g})$ and subsequently infected with S. marcescens at an MOI of 20 . CHI3L1 overexpression significantly enhanced the adhesion and invasion of S. marcescens on/into SW480 cells as compared with those of nontransfected cells. The adhesion and invasion assays were performed at least three times in separate experiments. ${ }^{\star} P<0.05$. (b) The effect of chitin, a GlcNAc polymer, on the adhesion efficiency of S. marcescens to SW480 cells was determined. Overnight pre-incubation of the bacteria or CECs with chitin was followed by an adhesion assay. S. marcescens significantly decreased their binding to CECS after incubating with chitin in a dose-dependent manner compared with untreated S. marcescens. In contrast, the adhesion of S. marcescens to SW480 cells was not inhibited when the epithelial cells instead of the bacteria were pretreated with chitin and the medium was removed before the addition of the inoculum to the cell monolayer. Each value is the mean \pm s.e.m. of at least three separate experiments. ${ }^{*} P<0.01$.

that endogenously express CHI3L1 were infected with all the three types of E. coli after 1 or $2 \mathrm{~h}$ of treatment with IPTG. Interestingly, WT CBP21-overexpressing E. coli significantly increased their adhesion rates to SW480 cells as compared with control- or lacZ-expressing E. coli (Figure 3b). However, the WT CBP21-overexpressing E. coli did not alter the rate of invasion into SW480 cells (data not shown) after 1 or $2 \mathrm{~h}$ of IPTG inductions (data not shown).

To further examine the involvement of CHI3L1 in the adhesion of WT CBP21-overexpressing E. coli to CECs, SW480 cells were transiently transfected with pCDNA4/ CHI3L1 expression vector and subsequently infected with WT CBP21 E. coli. After transfecting with the CHI3L1 vector, there was a significant increase in the number of WT CBP21 E. coli bound to SW480 cells compared with those of non- or control vector-transfected cells (Figure $3 \mathrm{~b}$ ). Interestingly, WT CBP21-overexpressing E. coli treated for $2 \mathrm{~h}$ with IPTG significantly increased adhesion to CHI3L1-overexpressing SW480 cells as compared with those treated for $1 \mathrm{~h}$ with IPTG (Figure 3c), suggesting that the adhesion of BL21 E. coli has been enhanced by the expression of CBP21 on the bacteria. These data suggest that bacterial CBP21 is involved in the adhesion but not invasion of E. coli to CECs, and CHI3L1 enhances the effect of CBP21-mediated bacterial adhesion to CECs.

\section{Inhibition of CBP21-Overexpressing E. coli Adhesion to CECs by Chitin}

To examine our hypothesis that bacteria bind to CECs through a CHI3L1/chitin complex, an effect of various carbohydrates on the adhesion efficiency of WT CBP21 E. coli to SW480 cells was determined. A duration of $1 \mathrm{~h}$ pre-incubation of the bacteria or SW480 cells with various carbohydrates was followed by an adhesion assay. Compatible with 


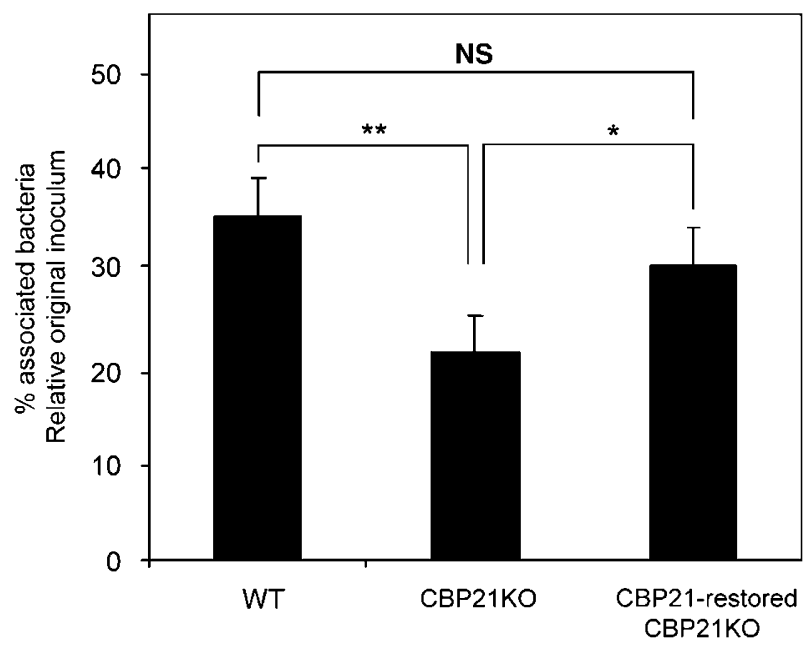

Figure 2 Effect of CBP21 expression in adhesion rate of $S$. marcescens in CECs. WT, CBP21 knockout and CBP21-restored CBP21 knockout strains of S. marcescens was used for bacterial adhesion assay. SW480 cells were infected with the S. marcescens for $2 \mathrm{~h}$ at an MOI of 20. CBP21 knockout strain of S. marcescens significantly decreased its adhesion to SW480 cells compared with WT strain of S. marcescens. In contrast, after restoring the CBP21 expression by merging CBP21 knockout strain of S. marcescens and CBP21-overexpressing PMMB67EH/HB101 E. coli, the bacteria significantly increased the adhesion rate compared with the CBP21 knockout strain of S. marcescens, but almost similar adhesion as WT strain of S. marcescens. Each value is the mean \pm s.e.m. Three separate experiments. NS, no significant difference, ${ }^{\star} P<0.05,{ }^{* *} P<0.01$.

the previous result (Figure 1b), WT CBP21 E. coli significantly decreased its binding to the cells after pre-incubating with chitin, compared with untreated WT CBP21 E. coli control (Figure 4a). In contrast, the adhesion of WT CBP21 E. coli to SW480 cells was not inhibited when the cells (instead of the bacteria) were pretreated with other carbohydrates (Figure $4 \mathrm{~b}$ ). These data suggest that chitin efficiently inhibits the adhesion of WT CBP21 E. coli to CECs, and WT CBP21 E. coli do not directly but indirectly bind to CHI3L1 on CECs. Therefore, the inhibitory effect of bacterial-epithelial interaction by chitin appears to be the result of the existence of endogenous chitin-like molecule(s) on CECs, and the complex of endogenous chitin-like molecule(s) and CHI3L1 on CECs may interact with the binding of CBP21 (on bacteria) and CHI3L1 (on CECs).

\section{Mutant Forms of CBP21-Overexpressing E. coli Significantly Decreased their Binding Ability}

Vaaje-Kolstad $e t a l^{21}$ revealed the crystal structure of CBP21 and showed that the roles of six highly conserved surfaceexposed residues (Y54, E55, E60, H114, D182 and N185) were important for the binding of CBP21 to chitin. To analyze the mechanism by which parts of CBP21 bind to the complex of CHI3L1/endogenous chitin-like molecule on CECs, we investigated the function of these conserved surface residues by mutating each of these residues to alanine (Y54A, E55A, E60A, H114A, D182A and N185A) of CBP21 in BL21AI strain of E. coli. Mutations in the $c b p$ genes did not affect the growth rates of these strains (data not shown). We performed adhesion assays by utilizing these mutant forms of CBP21-overexpressing E. coli and identified the critical region for the CBP21-mediated bacterial adhesion. Genetically engineered E. coli with a single mutation of either Y54 or E55 residue of CBP21 exhibited a significantly decreased binding to SW480 cells compared with WT CBP21 E. coli. The binding ability was $74 \%$ reduced by the combined mutations of three amino acids of Y54, E55 and E60 (3MU) compared with WT CBP21 E. coli (Figure 5). These results suggest that residues in the conserved surface area, especially Y54 and E55, are important for the ability of CBP21 to bind to the complex of CHI3L1/endogenous chitin-like molecule on CECs.

\section{Confocal Immunofluorescent Analysis Revealed That CBP21-Overexpressing Nonpathogenic E. coli Efficiently Bound to CHI3L1-Expressing Cells}

To confirm our results that WT of CBP21-overexpressing E. coli increased the adhesion rate to cell lines and mutant forms of CBP21-overexpressing E. coli decreased their binding ability, we next performed confocal immunofluorescent analysis by utilizing control vectors, including $E$. coli (control), WT of CBP21-overexpressing E. coli (WT-CBP21) and Y54, E55 and E60 point-mutated CBP21-overexpressing E. coli (3MU-CBP21). COS7 cells were transiently transfected with mouse CHI3L1 expression vector $(0.7 \mu \mathrm{g})$ and infected with control-, WT CBP21- or 3MU CBP21-overexpressing E. coli. As shown in Figure 6a, CHI3L1 was mainly expressed in perinuclear associated and surface membrane compartments. CBP21-overexpressing E. coli attached mainly to the surface membrane of the cells. We analyzed the number of bacterial adhesion per a single cell by counting more than 70 cells/ image in each group (control, WT-CBP21 or 3MU-CBP21). WT CBP21 E. coli significantly increased the adhesion to the CHI3L1-expressing COS7 cells compared with control E. coli (Figure 6b). Less number of 3MU-CBP21 E. coli bound to CHI3L1-expressing COS7 cells compared with the WT CBP21 E. coli (Figure 6b). Taken together, bacterial CBP21 is actively involved in the adhesion of E. coli to CHI3L1-overexpressing cells, and some amino-acid residues in the conserved surface area are important for the binding ability of CBP21.

\section{CHI3L1-Overexpression Enhanced the Adhesion Rate of CBP21-Overexpressing E. coli to CECs}

By utilizing the genetically engineered BL21 strain of E. coli, we next analyzed a role of CHI3L1 in the adhesion of CBP21overexpressing E. coli to CECs. SW480 cells were transiently transfected with pCDNA/CHI3L1 or mock (pCDNA4) vector and subsequently infected with control-, WT CBP21- or 3MU CBP21-overexpressing E. coli. Interestingly, enhanced CHI3L1 expression in SW480 cells significantly facilitated the adhesion of WT CBP21 E. coli to SW480 cells in a dosedependent manner compared with pCDNA mock, although 

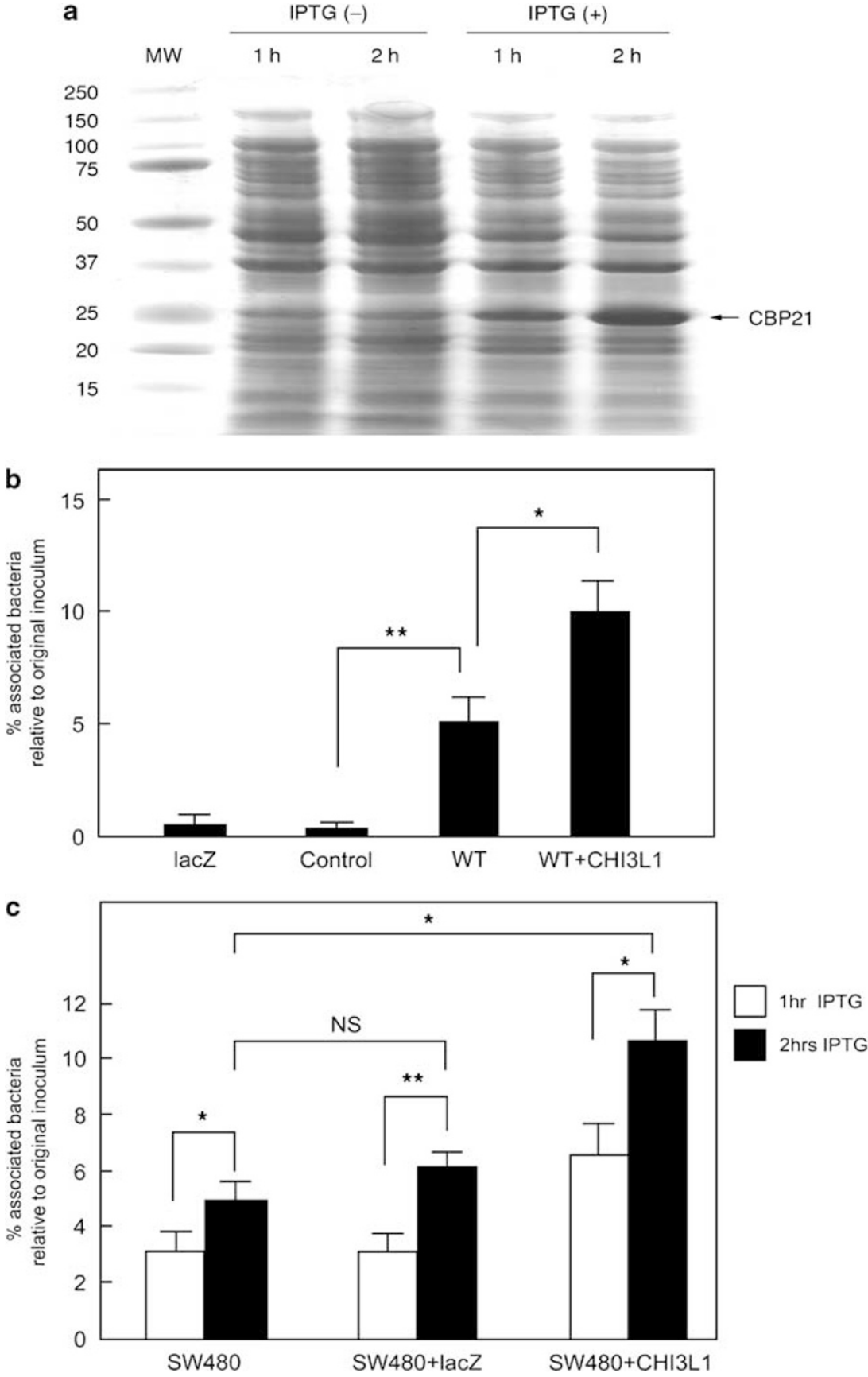

Figure 3 Generation of CBP-overexpressing E. coli and increased adhesion rate of the E. coli to CECs. (a) Recombinant CBP21 fusion protein was produced by BL21 strain of $E$. coli after 1 or $2 \mathrm{~h}$ incubation with $1 \mathrm{mM}$ IPTG and $0.2 \%$ L-arabinose. Bacterial pellet was collected, resuspended in PBS and subjected to freeze-thaw cycles four times. An equal volume of $2 \times$ SDS-polyacrylamide gel electrophoresis sample buffer was added to $100 \mu$ l of supernatant. A total of $20 \mu \mathrm{l}$ of samples were loaded on SDS-PAGE and stained with Coomassie blue. A total of $25 \mathrm{kDa}$ CBP21 fusion protein including tags was detected after the IPTG induction. (b) Increased adhesion of CBP21-overexpressing E. coli to CECs. Bacterial adhesion assays were performed after infecting with lacZ-pRSET (lacZ) vector-, pRSET empty (control) vector- or pRSET CBP21 WT (WT CBP21) vector-transformed BL21-Al strain of nonpathogenic E. coli. All groups of bacteria were cultured with $1 \mathrm{mM} \mathrm{IPTG}$ and $0.2 \%$ L-arabinose for $2 \mathrm{~h}$. SW480 cells were infected with each bacteria for $1 \mathrm{~h}$ at an MOI of 20. WT CBP21 E. coli significantly increased adhesion to SW480 cells. After transiently transfecting the CHI3L1 vector (0.7 $\mu \mathrm{g})$ to SW480 cells, there was a significant increase in the number of WT CBP21 E. coli bound to SW480 cells as compared with those of nontransfected cells. Each value is the mean \pm s.e.m. of at least three separate experiments. ${ }^{*} P<0.05,{ }^{*} P<<0.01$. (c) Increased adhesion of CBP21-overexpressing E. coli to CECs is IPTG dependent. Bacterial adhesion assays were performed after infecting with pRSET CBP21 WT (WT CBP21) vector-transformed BL21-Al strain of E. coli. The bacteria were cultured with $1 \mathrm{mM}$ IPTG and $0.2 \%$ L-arabinose for 1 or $2 \mathrm{~h}$. SW480 cells were transfected with empty pCDNA4 vector (control), lacZ-pCDNA4 vector (lacZ) or CHI3L1-pCDNA4 vector (CHI3L1) infected with each bacteria for $1 \mathrm{~h}$ at an MOI of 20. WT CBP21 E. coli significantly increased the adhesion to SW480 cells after $2 \mathrm{~h}$ incubation with IPTG compared with $1 \mathrm{~h}$ incubation in control-, LacZ- or CHI3L1-vector-transfected SW480 cells. Each value is the mean \pm s.e.m. of at least three separate experiments. ${ }^{\star} P<0.001,{ }^{* *} P<0.0001$. 
a

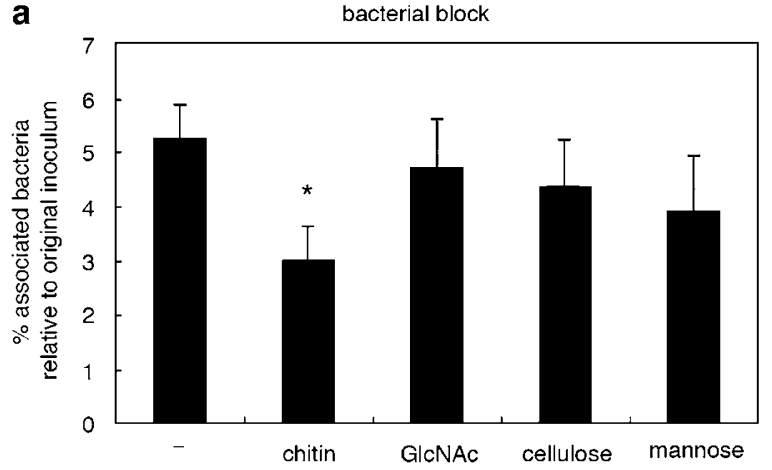

b

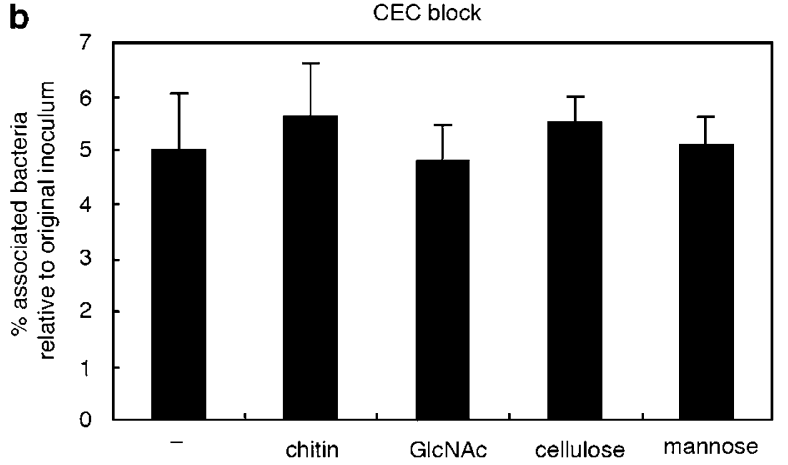

Figure 4 Effect of various carbohydrate pretreatments of CBP21-overexpressing E. coli on adhesion to CECs. (a) The effect of various carbohydrates on the adhesion efficiency of CBP21-overexpressing (WT CBP21) E. coli to SW480 cells was determined. Overnight pre-incubated bacteria with carbohydrates $(250 \mu \mathrm{g} / \mathrm{ml})$ were used for an adhesion assay. WT CBP21 E. coli significantly decreased its binding to CECs after pre-incubation with chitin compared with untreated control. Each value is the mean \pm s.e.m. of at least three separate experiments. ${ }^{*} P<0.05$.

(b) Pre-incubation of CECs with various carbohydrates did not change the adhesion of CBP21-overexpressing E. coli. Overnight pre-incubated SW480 cells with various carbohydrates $(250 \mu \mathrm{g} / \mathrm{ml})$ were used for an adhesion assay. The medium was removed before the addition of the inoculum to the CECs monolayer. The adhesion of WT CBP21 E. coli to SW480 cells was not inhibited when CECs were pre-incubated with various carbohydrates. Each value is the mean \pm s.e.m. of at least three separate experiments.

the adhesion of control and 3MU CBP21 E. coli were not changed even after the CHI3L1 overexpression (Figure 7a).

To confirm whether the enhanced adhesion of CBP21overexpressing bacteria to CECs is mediated by CHI3L1, studies to inhibit CHI3L1 expression and function were performed. To block the CHI3L1 activity, we utilized antiCHI3L1 Ab, which previously confirmed the blocking activity against CHI3L1 protein. ${ }^{18}$ The CHI3L1 Ab was added $1 \mathrm{~h}$ before the infection with WT CBP21- or 3MU CBP21-overexpressing E. coli in $\mathrm{CHI} 3 \mathrm{~L} 1$ expression vector-transfected SW480 cells. As a result, pretreatment with anti-CHI3L1 Ab significantly reduced the adhesion of WT CBP21 E. coli to the SW480 cells (Figure 7b). In addition, a dose-dependent effect of the anti-CHI3L1 Ab treatment on the inhibition was observed. In contrast, the same concentration of control normal rabbit IgG did not affect the bacterial adhesion. The anti-CHI3L1 Ab treatment did not significantly suppress the

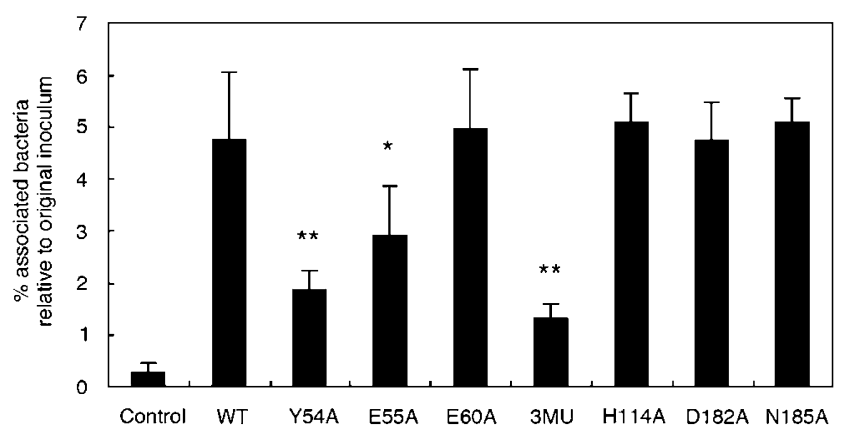

Figure 5 Generation of point mutant forms of CBP21-overexpressing $E$. coli and adhesion rate of the E. coli to CECs. Adhesion assays were performed after infecting with pRSET empty (control) vector-, pRSET CBP21 WT (WT CBP21) vector-, pRSET CBP21 mutant forms of six residues to alanine (Y54A, E55A, E60A, H114A, D182A and N185A) vectoror pRSET CBP21 3 combined mutant form of Y54A, E55A and E60A (3MU) vector-transformed BL21-Al strain of E. coli. All groups of bacteria were cultured with $1 \mathrm{mM} \mathrm{IPTG}$ and $0.2 \%$ L-arabinose for $2 \mathrm{~h}$. BL21 strain of E. coli with a single mutation of either Y54 or E55 residue of CBP21 exhibited a significantly decreased binding to SW480 cells compared with WT CBP21 E. coli. The binding ability was $74 \%$ reduced by the $3 \mathrm{MU}$ compared with WT CBP21 E. coli. Each value is the mean \pm s.e.m. of at least three separate experiments. ${ }^{*} P<0.05,{ }^{*} P<0.01$.

adhesion of 3MU CBP21 E. coli (Figure 7b). These results strongly suggest that CHI3L1 enhances the CBP21-overexpressing E. coli to CECs through the conserved amino-acid region on CBP21.

\section{Inhibition of CHI3L1 by siRNA Reduced the Adhesion Rate of CBP21-Overexpressing BI21 E. coli to CECs}

RNA interference is an effective method to knock down specific gene expressions. ${ }^{26}$ By utilizing this siRNA technology, the functional role of CHI3L1 in the adhesion of CBP21overexpressing E. coli to CECs was examined. CMT93 cells that endogenously express $\mathrm{CHI} 3 \mathrm{~L} 1$ were transiently transfected with three different kinds of CHI3L1 siRNAs or control siRNAs. As shown in Figure 8a, CHI3L1 siRNAs significantly suppress the protein expression of endogenous CHI3L1 compared with control siRNAs. Inhibition with combined CHI3L1 siRNAs (no. 1, no. 2 and no. 3 siRNAs) significantly $(P<0.01)$ knocks down the CHI3L1 expression as both message ${ }^{18}$ and protein (Figure 8a) levels, and significant fewer numbers of WT-CBP21 E. coli attached on CMT93 cells as compared with control siRNA (Figure 8b). In contrast, 3MU CBP21 E. coli did not show significant reduction in the number of the adhered bacteria to CECs compared with control E. coli (Figure 8c). Taken together, the series of these findings clearly show that CHI3L1 possesses the ability to facilitate the bacterial adhesion to CECs through the conserved amino-acid region of bacterial CBP21 and presumably its homologs.

\section{DISCUSSION}

As we demonstrated previously, CHI3L1, a member of the mammalian chitinase family, enhances pathogenic 
and potentially pathogenic bacterial adhesion and invasion on/into CECs. ${ }^{18}$ In contrast to pathogenic and potentially pathogenic bacteria, CHI3L1 does not affect the adhesion and invasion of nonpathogenic E. coli such as DH10B and $\mathrm{DH} 5 \alpha$ strains to CEC. ${ }^{18}$ There is an increasing evidence that the mucosa-associated flora may be important in the pathogenesis of inflammatory bowel disease (IBD). ${ }^{27}$ In patients with IBD, especially CD patients, a thick bacterial film (so called biofilm) was observed tightly attached to the mucosal surface, but not in control individuals who have self-limiting colitis. ${ }^{28}$ It is unclear how these commensal bacteria strongly and continuously bind to CEC in IBD patients. Characterization and identification of key molecule(s), which will regulate commensal bacterial adhesion and invasion on/ into CECs, are crucial to understand the initiation, exacerbation and perpetuation of IBD. In this study, we have demonstrated that $S$. marcescens significantly increased their adhesion to CECs after CHI3L1 overexpression. CHI3L1 has no chitinase activity, ${ }^{3}$ but still possesses a binding ability to chitin and chitooligosaccharides with high affinity through a preserved hydrophobic substrate binding cleft, ${ }^{19,16}$ and the majority of chitinase-producing pathogenic bacteria have the ability to produce chitin-binding molecules such as CBP $21 .^{22}$ We found that CBP21 knockout strain of $S$. marcescens significantly reduced in the adhesion but not invasion rate to CECs. Therefore, CBP21 seems to be involved in the bacterial adhesion to CECs, and CHI3L1 may bind to bacteria through CBPs.

To investigate our hypothesis that bacteria would bind to CECs through a CHI3L1/chitin/CBP complex, bacteria or CECs were pretreated with various carbohydrates and followed by adhesion assays. Interestingly, not only $S$. marcescens but also CBP21-overexpressing nonpathogenic E. coli significantly decreased their binding to CECs after pretreatment of these bacteria with chitin, compared with untreated control. In contrast, the adhesion of $S$. marcescens and CBP21-overexpressing E. coli to CECs were not inhibited when CECs instead of the bacteria were pretreated with various carbohydrates. These results suggest a possibility that bacterial CBP21 does not directly bind to CHI3L1 on CECs, but presumably binds to CECs via endogenous chitin-like molecules. As the candidate of endogenous chitin-like molecule, we speculate that endogenous polysaccharide (glycan) structure on the surface of CECs would be the most probable component, and the complex is likely to bind with CBP-expressing bacteria. Although chitin is not present in mammalian cells, the GlcNAc, chitin monomer, is a common modification of glycoproteins and lipids present on the intestinal epithelium of mammals. ${ }^{29,30}$ It has also been demonstrated that the presence of a GlcNAc residue alone is not sufficient for inhibiting of bacterial binding to CECs, and additional carbohydrate residues are required for high-affinity adherence. $^{31}$

To further examine the functional role of CBPs in bacterial adhesion, we generated CBP21-overexpressing BL21-AI strain of E. coli. CBP21-overexpressing (WT CBP21) E. coli significantly increased the adhesion to SW480 cells by binding assay, although BL21-AI strain of E. coli and control lacZ-overexpressing E. coli did not attach to SW480 cells. In addition, CHI3L1 expression vector-transfected SW480 cells significantly increased the adhesion of WT-CBP21 E. coli to SW480 cells as compared with those of nontransfected cells. These data suggest that CBP21 is involved in the bacterial adhesion to CECs, and CHI3L1 enhances the CBP21-mediated bacterial adhesion to CECs.

The crystal structures of CBP21 show that six highly conserved surface-exposed residues (Thy-54, Glu-55, Glu-60, His-114, Asp-182 and Asn-185) play an important role in the binding of CBP21 to chitin. ${ }^{21}$ To analyze the mechanism by which parts of CBP21 actually binds to endogenous chitinlike molecules, we generated mutant forms of CBP21 E. coli by mutating each of these six amino-acid residues to alanine. As a result, genetically engineered $E$. coli with a single mutation of either Thy-54 or Glu-55 residue of CBP21 exhibited a decreased binding ability to SW480 cells compared with WT CBP21 E. coli. The binding ability was $74 \%$ reduced by the combined mutations of all the three amino acids of Thy54, Glu-55 and Glu-60 (3MU) compared with WT. These results strongly suggest that residues in the conserved surface area, especially Tyr-54 and Glu-55, are important for the ability of CBP21 to bind to chitin or endogenous chitin-like molecules, which may be expressed on CECs. It has been well analyzed by Vaaje-Kolstad et $\mathrm{al}^{21}$ that the binding curve between CBP21 and chitin was decreased largely in point mutants of Y54A and E60A. Thy-54 is the only one conserved aromatic residue that is fully exposed on the surface of CBP21 and is essential to generate the tyrosine ring for binding with chitin. ${ }^{21,32}$ These findings are consistent with our results that the mutation in Thy-54, Glu-55 and Glu-60 affects the function of CBP21 in bacterial adhesion to CECs and the binding may be mediated by the complex of putative chitin-like endogenous glycan and CHI3L1. Our result that Y54A and E55A exhibit significantly decreased binding ability to CECs does not correlate completely with the result that E55A is less impaired than E60A in $\mathrm{Kd}$ for binding to $\beta$-chitin described by Vaaje-Kolstad et al. Mutational effects that were observed in our results and in the study of Vaaje-Kolstad et al may be explained by assuming that certain residues are of particular importance for binding to certain subtypes of chitin or certain parts of chitin-like molecules. Confocal immunofluorescent analysis also reveals that WT CBP21-overexpressing BL21 E. coli, but not control BL21 E. coli, efficiently binds to CHI3L1-expressing cells. Taken together, bacterial CBP21 is actively involved in the adhesion to CHI3L1-expressing cells, and residues in the conserved surface area of CBP21 are important for the binding ability of CBP21 to the complex of CHI3L1/endogenous chitin-like molecule on epithelial cells.

To further investigate a biological significance of CHI3L1 in the interaction of bacterial CBP21 and CECs, we transiently transfected CHI3L1 expression vector on CECs 
followed by infecting the cells with WT CBP21 E. coli. Interestingly, transfection with CHI3L1 expression vector to CECs significantly facilitated the adhesion of WT CBP21 E. coli in a dose-dependent manner compared with mock-transfected CECs. To confirm whether the enhanced adhesion of CBP21-expressing bacteria to CECs is mediated by CHI3L1, we performed blocking experiments by utilizing anti-CHI3L1-specific Ab and CHI3L1 siRNA, the effects of a

CHI3L1

E. coli
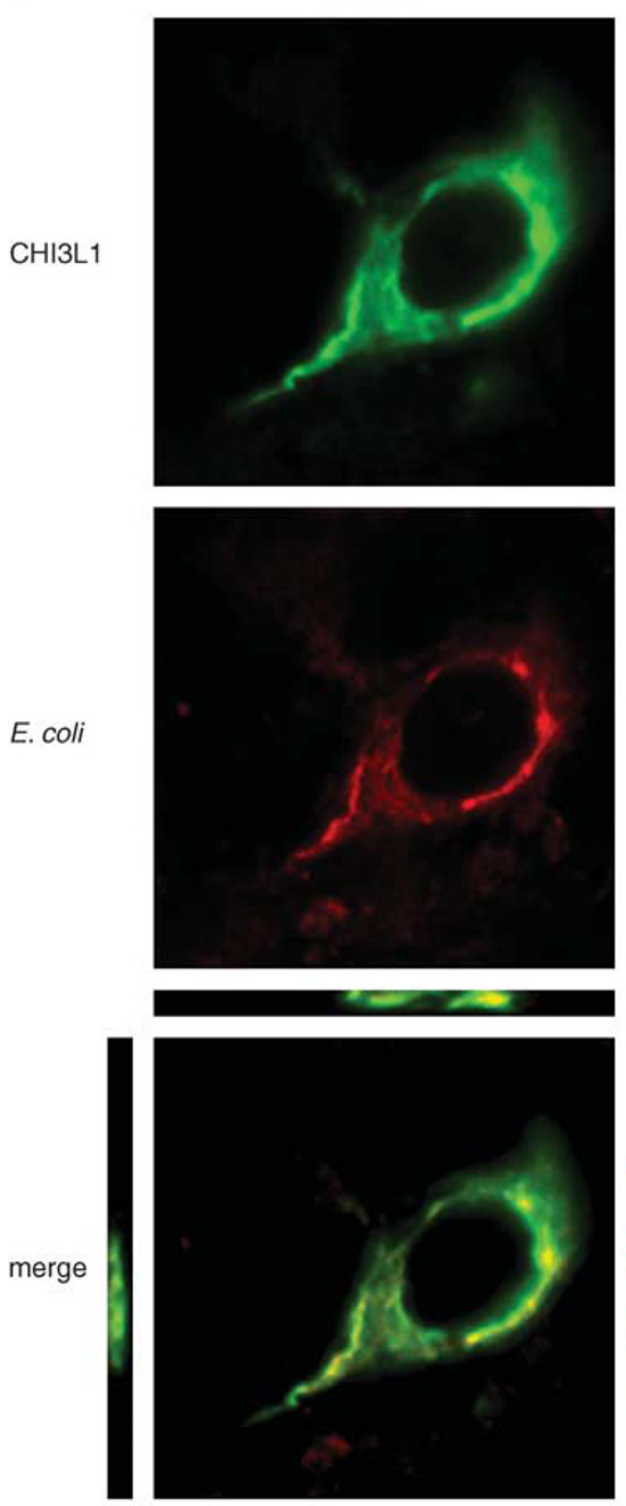

WT
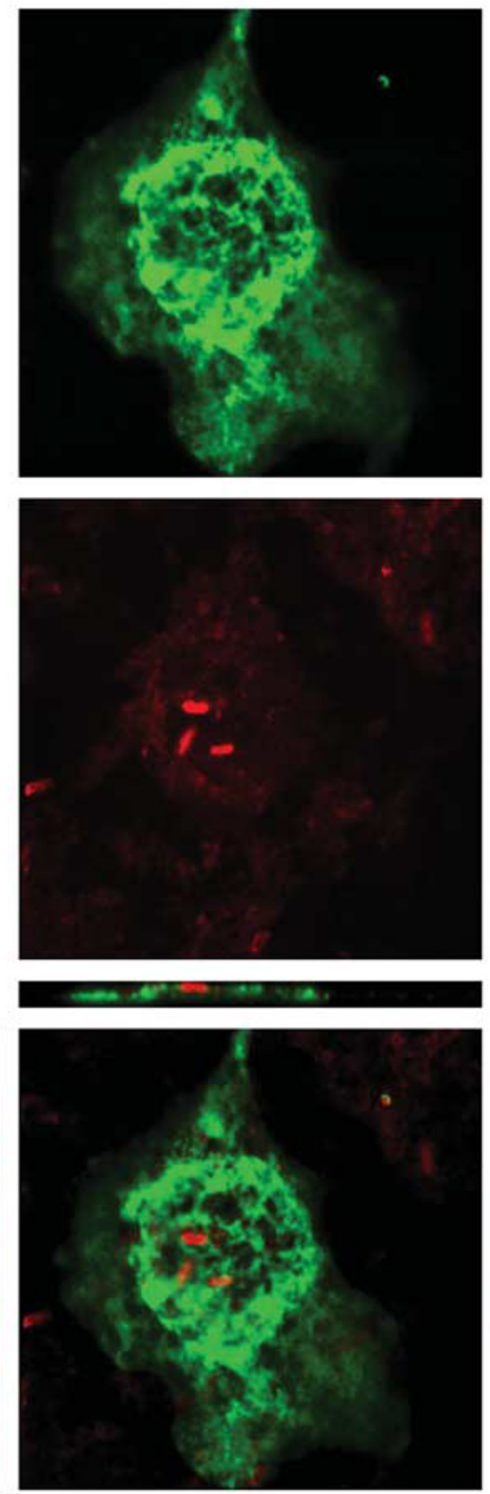

$3 \mathrm{MU}$
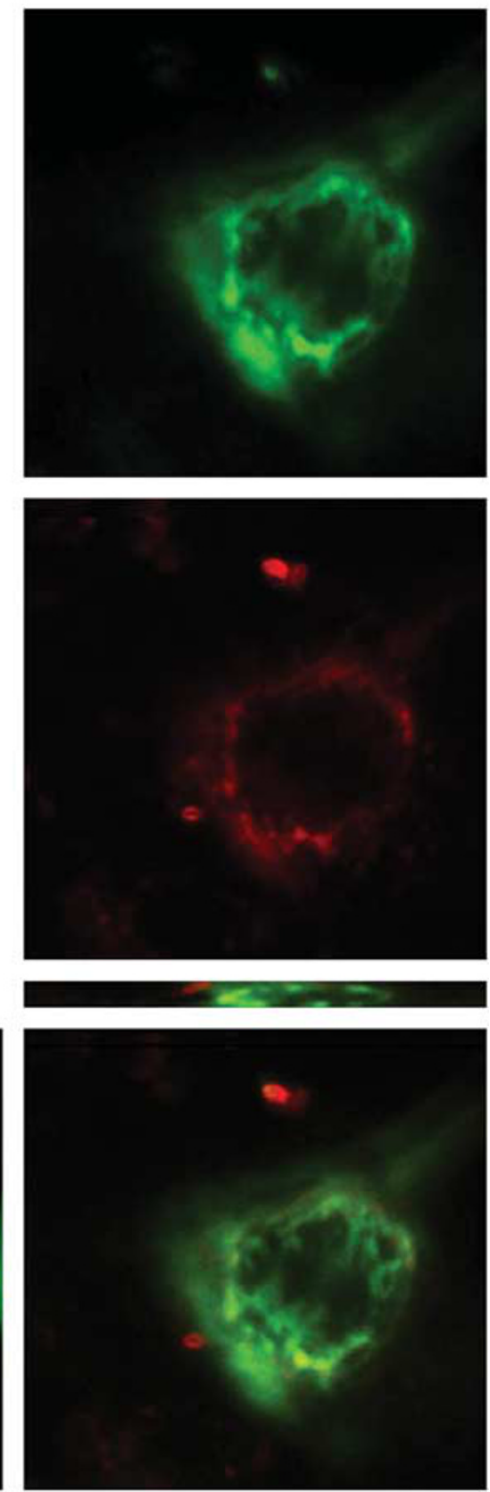

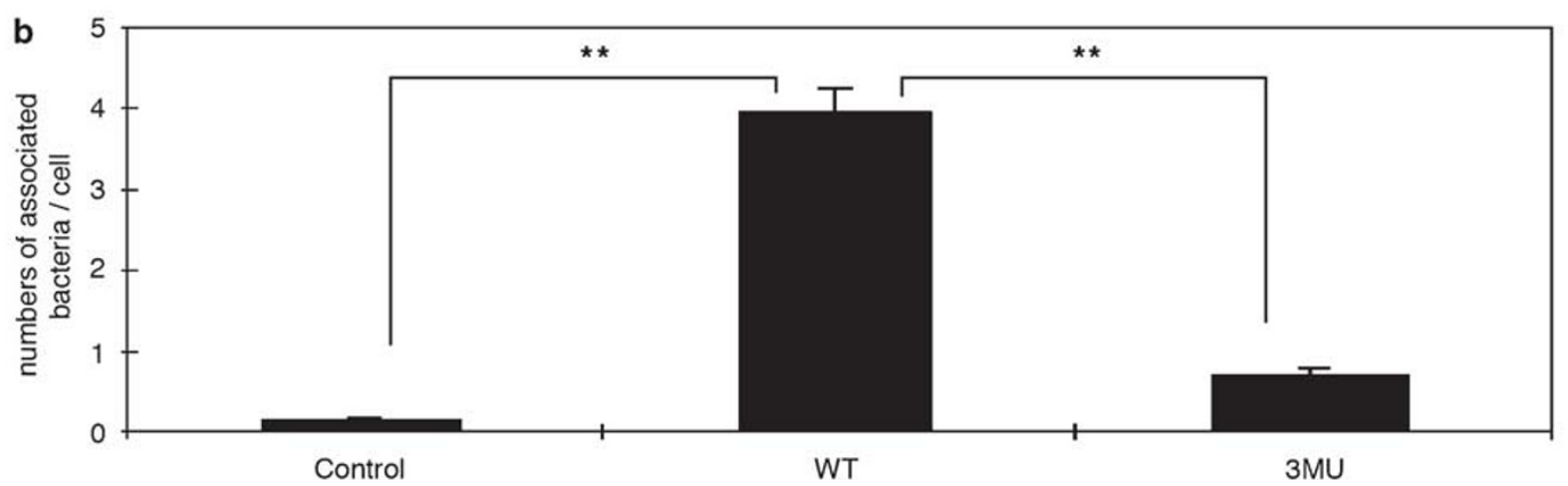


which were already confirmed in the previous studies. The neutralization of CHI3L1 by pre-incubating with anti-CHI3L1 Ab significantly reduced the adhesion of WT
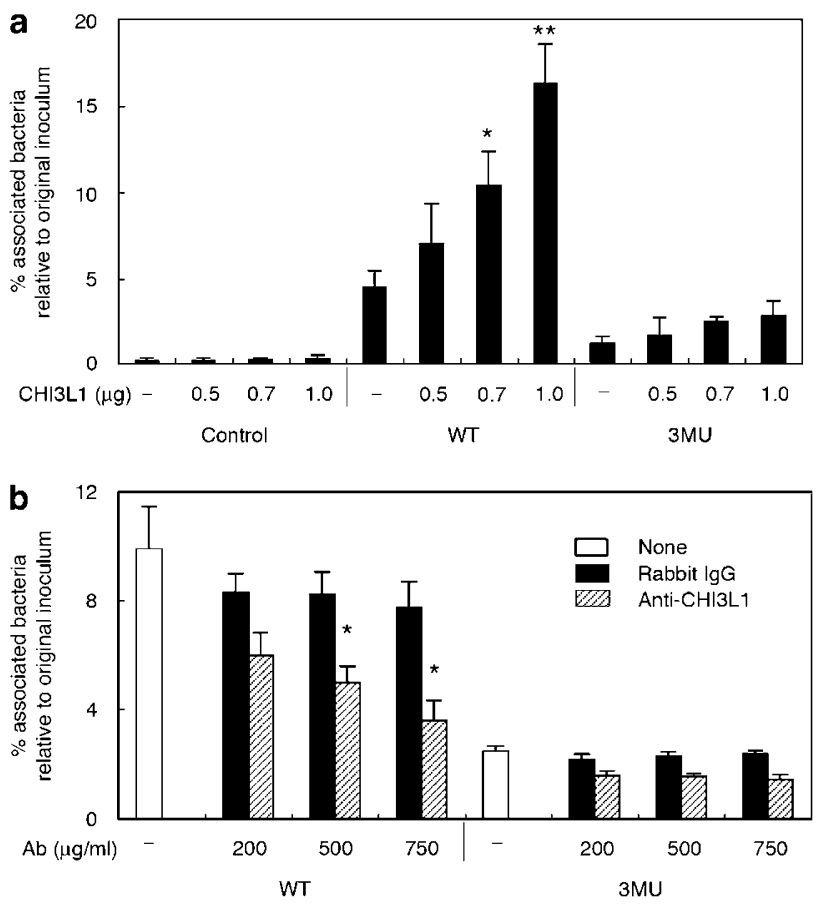

Figure 7 Effect of three amino acid mutations (3MU) in CBP21overexpressing $E$. coli on adhesion to CECs. (a) CHI3L1 overexpression significantly enhanced the adhesion of WT CBP21-overexpressing E. coli to CECs SW480 cells, which endogenously express CHI3L1, were transiently transfected with pCDNA4/CHI3L1 or mock pCDNA4 vector and subsequently infected with control-, WT- and 3MU CBP21-overexpressing E. coli for $1 \mathrm{~h}$ at an MOI of 20. CHI3L1 overexpression in SW480 cells significantly facilitated WT CBP21 E. coli in a dose-dependent manner compared with pCDNA mock. In contrast, the adhesion of control and $3 \mathrm{MU}$ CBP21 E. coli did not change the adhesion to CHI3L1-overexpressing SW480 cells. Each value is the mean \pm s.e.m. of at least three separate experiments. ${ }^{*} P<0.05,{ }^{*} P<0.01$. (b) Anti-CHI3L1 Ab pretreatment reduced the adhesion of WT CBP21-overexpressing $E$. coli to CECs. Neutralization of CHI3L1 by pre-incubation with anti-CHI3L1 Ab was performed in pCDNA4/CHI3L1 $(0.7 \mu \mathrm{g})$-transfected SW480 cells. The anti-CHI3L1 Ab pretreatment (hatched bars) significantly reduced the WT CBP21 E. coli adhesion in a dose-dependent manner. In contrast, control rabbit IgG (closed bars) did not affect the bacterial adhesion to the CECs. The anti-CHI3L1 Ab pretreatment did not significantly suppress the adhesion of 3MU CBP21 E. coli. Each value is the mean \pm s.e.m. of at least three separate experiments. ${ }^{*} P<0.05$.
CBP21 E. coli to the CHI3L1-expressing CECs in a dose-dependent manner. Inhibition of endogenously expressed CHI3L1 by mouse CHI3L1-specific siRNA also significantly reduced the adhesion of WT CBP21 E. coli to CECs. Taken together, the series of these findings clearly demonstrate that $\mathrm{CHI} 3 \mathrm{~L} 1$ plays a critical role in the adhesion of CBP-expressing bacteria to CECs.

It has been elegantly described that a putative CBP, GbpA (GlcNAc-binding protein), which is produced by Vibrio cholerae, significantly enhances the bacterial adhesion to both epithelial cell surfaces and chitin, most probably by a direct interaction with GlcNAc residues expressing on epithelial cells. ${ }^{33}$ Interestingly, both GbpA and CBP21 belong to CBM (carbohydrate-binding module) family 33 and have multiple alignments in the sequences. Both proteins contain chitinbinding type-3 domain (pfam03067), and therefore CBP21 may interact with GlcNAc residues on CECs simliar to GbpA. Both GbpA and CBP21 have been reported as a secreted type of protein. ${ }^{20,33}$ However, the exact secretion system of both proteins still remains unknown. As GbpA was detected at periplasmic and culture supernatant, ${ }^{33}$ we consider that CBP21 may also be present in two forms, a secreted form and a membrane-associated form. It is speculated that the two forms of CBP21 may interact with each other to promote bacterial attachment to CECs. Structurally conserved chitinbinding properties exist in distantly related microorganisms, such as Streptomyces, Serratia, Yersinia and Vibrio, suggesting a wide distribution of this type of CBPs in chitinolytic microorganisms. ${ }^{20}$ Specific adhesion of mucosa-associated bacteria in IBD and colon cancer might be enhanced by the inducible expression of mucosal glycol conjugates on CECs. ${ }^{34}$ In fact, CHI3L1 is expressed on CECs only under intestinal inflammatory conditions, but not quiescent IBD or normal epithelial cells. ${ }^{18}$ We speculate that CHI3L1 may be expressed on CECs only under the intestinal inflammatory conditions and subsequently binds to endogenous chitin-like molecules on the epithelial surface, and therefore the attachment of CBP-expressing bacteria to CECs is enhanced. Further studies would be required to clarify the existence of mucosal glycoconjugates (presumably chitin-like glycans), which may generate complex formation with CHI3L1 on CECs under the inflammatory conditions. In conclusion, the results obtained in this study demonstrate that CBP21 and its homologs may be required for the CHI3L1-mediated

Figure 6 Confocal immunofluorescent analysis of CBP-overexpressing E. coli bound to CHI3L1 expressing cells. (a) COS7 cells were transiently transfected with mouse CHI3L1 expression vector $(0.7 \mu \mathrm{g})$ for $24 \mathrm{~h}$ and infected with control-, WT CBP21- and 3MU (Y54A, E55A and E60A) CBP21-overexpressing BL21 strain of E. coli for $1 \mathrm{~h}$ at an MOI of 20. Cells were stained with mouse anti-X-press monoclonal Ab followed by FITC-conjugated anti-mouse IgG. E. coli were stained with rabbit anti-E. coli polyclonal Ab followed by rhodamine-conjugated anti-rabbit lgG. CHI3L1 (green) was mainly expressed in perinuclear, cytoplasmic and surface membrane compartments. CBP21-overexpressing E. coli (red) attached to the surface membrane of the cells. The $x z$ (the one on the top) and $y z$ (the one on the left) cross sections are shown in the merged images. (b) The number of bacterial adhesion per CHI3L1-expressing cell was counted to be more than 70 cells for all the three groups (control, WT, 3MU). CBP21-overexpressing (WT CBP21) E. coli significantly increased the adhesion to CHI3L1-expressing cells compared with control E. coli. Less number of Y54A, E55A and E60A mutated CBP21-overexpressing (3MU CBP21) E. coli bound to CHI3L1-expressing cells as compared with WT CBP21 E. coli. Each value is the mean \pm s.e.m. ${ }^{* *}>0.01$. 

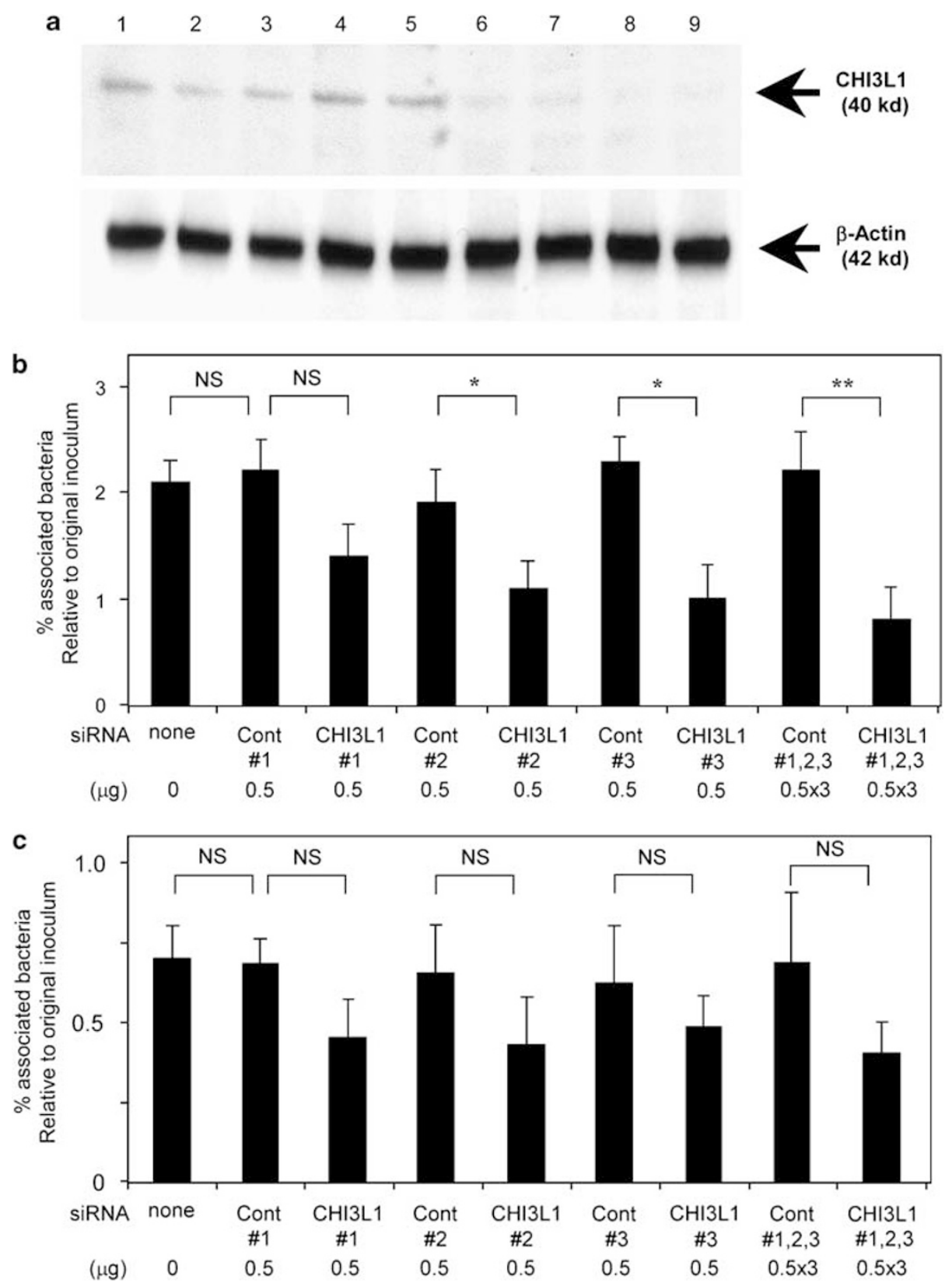

Figure 8 Effect of CHI3L1 siRNAs in adhesion of WT CBP21-overexpressing E. coli to CECs. (a) CMT93 cells that endogenously express CHI3L1 were seeded at a density of $1 \times 10^{5}$ cells per well. CMT93 cells were transiently transfected with three different kinds of mouse CHI3L1 or control siRNAs for $48 \mathrm{~h}$. Protein expressions of $\mathrm{CHI} 3 \mathrm{~L} 1$ and $\beta$-actin were analyzed by western blot analysis. Protein expressions of $\mathrm{CHI} 3 \mathrm{~L} 1$ and $\beta$-actin were detected in the protein samples of CMT93 cells, which were transfected without siRNA (lane 1) or transfected with control siRNA no. 1 (lane 2), control siRNA no. 2 (lane 3), control siRNA no. 3 (lane 4), control siRNA nos.1-3 (lane 5), CHI3L1 siRNA no. 1 (lane 6), CHI3L1 siRNA no. 2 (lane 7), CHI3L1 siRNA no. 3 (lane 8) or CHI3L1 siRNA nos. 1-3 (lane 9). CHI3L1 siRNA transfection significantly reduced the CHI3L1 protein expression as compared with the control siRNA transfection. (b) CMT93 cells were transfected with either each or combined CHI3L1 siRNAs or control siRNAs. Transfected cells with CHI3L1 siRNA no. 2 only, no. 3 only or all the three were adhered with significantly less number of WT CBP21 E. coli compared with control siRNAs-treated groups. Each value is the mean \pm s.e.m. of three separate experiments. NS, no significant difference, ${ }^{\star} P<0.05, * \star P<0.01$. (c) After being infected with $3 \mathrm{MU}$ CBP21 E. coli for $1 \mathrm{~h}$ at an MOI of 20, none of each or combined CHI3L1-specific siRNAs did not alter the adhesion to CMT93 cells compared with control siRNAs. Each value is the mean \pm s.e.m. of at least three separate experiments. NS, no significant difference.

enhancement of potentially pathogenic bacterial adhesion to epithelial cells through the conserved amino-acid residues. Both CHI3L1 and CBPs may play an important as well as complicated role in the commensal bacterial adhesion to the CECs under the inflammatory conditions. Blockade of CHI3L1 and/or CBPs would be an effective therapeutic strategy for the treatment of IBD in the future. 


\section{ACKNOWLEDGEMENTS}

We are grateful to Drs Daniel K Podolsky, Cathy R Nagler, Hans-Christian Reinecker, Ramnik Xavier, Scott B Snapper and Atsushi Mizoguchi for their helpful discussions. We thank Dr Masanobu Nanno (Yakult Central Institute for Microbiological Research, Tokyo, Japan) and Mr David Adamovich (GI Unit, Massachusetts General Hospital) for their helpful advices and discussions, Dr Kazushi Suzuki and Mr Yasuhiro Fujiu for constructing CBP21 mutant strain of S. marcescens, and Dr Carmen Alonso (GI Unit,

Massachusetts General Hospital) for confocal microscopic analysis. We also thank Ms Sarah Murphy for her excellent secretarial assistance in preparing this manuscript and Ms Naomi Tsuru for her technical assistance. This work has been supported by National Institute of Health (DK64289, DK74454 and DK43351) and grants from the Eli and Edythe L Broad Medical Foundation and American Gastroenterological Association Foundation to EM.

1. Debono $M$, Gordee RS. Antibiotics that inhibit fungal cell wall development. Annu Rev Microbiol 1994;48:471-497.

2. Shahabuddin M, Toyoshima T, Aikawa $M$, et al. Transmission-blocking activity of a chitinase inhibitor and activation of malarial parasite chitinase by mosquito protease. Proc Natl Acad Sci USA 1993;90: 4266-4270.

3. Hakala BE, White $C$, Recklies AD. Human cartilage gp-39, a major secretory product of articular chondrocytes and synovial cells, is a mammalian member of a chitinase protein family. J Biol Chem 1993;268:25803-25810.

4. Boot RG, Renkema GH, Strijland A, et al. Cloning of a cDNA encoding chitotriosidase, a human chitinase produced by macrophages. J Biol Chem 1995;270:26252-26256.

5. Hu B, Trinh K, Figueira WF, et al. Isolation and sequence of a novel human chondrocyte protein related to mammalian members of the chitinase protein family. J Biol Chem 1996;271:19415-19420.

6. Jin HM, Copeland NG, Gilbert DJ, et al. Genetic characterization of the murine $\mathrm{Ym} 1$ gene and identification of a cluster of highly homologous genes. Genomics 1998;54:316-322.

7. Boot RG, Blommaart EF, Swart $E$, et al. Identification of a novel acidic mammalian chitinase distinct from chitotriosidase. J Biol Chem 2001;276:6770-6778.

8. Buhi WC. Characterization and biological roles of oviduct-specific oestrogen-dependent glycoprotein. Reproduction 2002;123:355-362.

9. Kzhyshkowska J, Mamidi S, Gratchev A, et al. Novel stabilin-1 interacting chitinase-like protein (SI-CLP) is up-regulated in alternatively activated macrophages and secreted via lysosomal pathway. Blood 2006;107:3221-3228.

10. Johansen JS, Williamson MK, Rice JS, et al. Identification of proteins secreted by human osteoblastic cells in culture. J Bone Miner Res 1992;7:501-512.

11. Rehli M, Krause SW, Andreesen R. Molecular characterization of the gene for human cartilage gp-39 (CHI3L1), a member of the chitinase protein family and marker for late stages of macrophage differentiation. Genomics 1997:43:221-225.

12. Nyirkos $\mathrm{P}$, Golds EE. Human synovial cells secrete a $39 \mathrm{kDa}$ protein similar to a bovine mammary protein expressed during the nonlactating period. Biochem J 1990;269:265-268.

13. Johansen JS, Jensen HS, Price PA. A new biochemical marker for joint injury. Analysis of YKL-40 in serum and synovial fluid. $\mathrm{Br} J$ Rheumatol 1993;32:949-955.

14. Shackelton LM, Mann DM, Millis AJ. Identification of a 38-kDa heparinbinding glycoprotein (gp38k) in differentiating vascular smooth muscle cells as a member of a group of proteins associated with tissue remodeling. J Biol Chem 1995;270:13076-13083.
15. Krause SW, Rehli M, Kreutz M, et al. Differential screening identifies genetic markers of monocyte to macrophage maturation. J Leukoc Biol 1996:60:540-545.

16. Renkema GH, Boot RG, Au FL, et al. Chitotriosidase, a chitinase, and the 39-kDa human cartilage glycoprotein, a chitin-binding lectin, are homologues of family 18 glycosyl hydrolases secreted by human macrophages. Eur J Biochem 1998;251:504-509.

17. Volck B, Price PA, Johansen JS, et al. YKL-40, a mammalian member of the chitinase family, is a matrix protein of specific granules in human neutrophils. Proc Assoc Am Physicians 1998; 110:351-360.

18. Mizoguchi E. Chitinase 3-like-1 exacerbates intestinal inflammation by enhancing bacterial adhesion and invasion in colonic epithelial cells. Gastroenterology 2006;130:398-411.

19. Fusetti F, Pijning T, Kalk KH, et al. Crystal structure and carbohydratebinding properties of the human cartilage glycoprotein-39. J Biol Chem 2003;278:37753-37760.

20. Suzuki K, Suzuki M, Taiyoji M, et al. Chitin binding protein (CBP21) in the culture supernatant of Serratia marcescens 2170. Biosci Biotechnol Biochem 1998;62:128-135.

21. Vaaje-Kolstad G, Houston DR, Riemen AH, et al. Crystal structure and binding properties of the Serratia marcescens chitin-binding protein CBP21. J Biol Chem 2005;280:11313-11319.

22. Vaaje-Kolstad G, Horn SJ, van Aalten DM, et al. The non-catalytic chitin-binding protein CBP21 from Serratia marcescens is essential for chitin degradation. J Biol Chem 2005;280:28492-28497.

23. Suzuki K, Uchiyama T, Suzuki M, et al. LysR-type transcriptional regulator ChiR is essential for production of all chitinases and a chitinbinding protein, CBP21, in Serratia marcescens 2170. Biosci Biotechnol Biochem 2001;65:338-347.

24. Hertle R, Schwarz H. Serratia marcescens internalization and replication in human bladder epithelial cells. BMC Infect Dis 2004;4:16.

25. Zeltins A, Schrempf H. Specific interaction of the Streptomyces chitin-binding protein CHB1 with $\alpha$-chitin - the role of individual tryptophan residues. Eur J Biochem 1997;246:557-564.

26. McManus MT. Small RNAs and immunity. Immunity 2004;21: 747-756.

27. Strober W, Fuss I, Mannon P. The fundamental basis of inflammatory bowel disease. J Clin Invest 2007;117:514-521.

28. Swidsinski A, Ladhoff A, Pernthaler A, et al. Mucosal flora in inflammatory bowel disease. Gastroenterology 2002;122: 44-54.

29. Bjork S, Breimer ME, Hansson GC, et al. Structures of blood group glycosphingolipids of human small intestine. A relation between the expression of fucolipids of epithelial cells and the ABO, Le and Se phenotype of the donor. J Biol Chem 1987;262: 6758-6765.

30. Finne J, Breimer ME, Hansson $\mathrm{GC}$, et al. Novel polyfucosylated $N$-linked glycopeptides with blood group $A, H, X$, and $Y$ determinants from human small intestinal epithelial cells. J Biol Chem 1989;264:57205735.

31. Fumagalli $\mathrm{O}$, Tall $\mathrm{BD}$, Schipper $\mathrm{C}$, et al. $\mathrm{N}$-glycosylated proteins are involved in efficient internalization of Klebsiella pneumoniae by cultured human epithelial cells. Infect Immun 1997;65:4445-4451.

32. Henrissat B, Davies G. Structural and sequence-based classification of glycoside hydrolases. Curr Opin Struct Biol 1997;7:637-644.

33. Kirn TJ, Jude BA, Taylor RK. A colonization factor links Vibrio cholerae environmental survival and human infection. Nature 2005;438: 863-866.

34. Rhodes JM. Unifying hypothesis for inflammatory bowel disease and associated colon cancer: sticking the pieces together with sugar. Lancet 1996;347:40-44. 